\title{
Voyage au cœur des pratiques punitives
}

Introduction

Introduction. Journey to the heart of punitive practices

Introducción. Viaje en el corazón de las prácticas punitivas

\section{Eirick Prairat}

\section{(2) OpenEdition Journals}

Édition électronique

URL : https://journals.openedition.org/ries/8598

DOI : $10.4000 /$ ries. 8598

ISSN : 2261-4265

\section{Éditeur}

France Education international

\section{Édition imprimée}

Date de publication : 1 septembre 2019

Pagination : 29-36

ISBN : 978-2-85420-624-1

ISSN : $1254-4590$

\section{Référence électronique}

Eirick Prairat, "Voyage au cœur des pratiques punitives ", Revue internationale d'éducation de Sèvres [En ligne], 81 | septembre 2019, mis en ligne le 01 septembre 2019, consulté le 13 octobre 2021. URL: http://journals.openedition.org/ries/8598; DOI : https://doi.org/10.4000/ries.8598 


\title{
La sanction en éducation
}

\section{Introduction}

Voyage au cœur des pratiques punitives

\author{
Eirick Prairat
}

Université de Lorraine

C'est un beau voyage qui est proposé aux lecteurs. De l'Afrique de l'Ouest à la Russie, de l'Amérique du Nord au Japon, ce numéro consacré à la question de la sanction nous fait découvrir des paysages éducatifs qui se ressemblent, tout en étant très différents. Disons-le d'entrée, nous avons eu beaucoup de mal à réunir un ensemble de contributeurs. Il est vrai que cette question de la sanction a été frappée, ces dernières années, d'indignité intellectuelle dans les pays européens et nord-américains. Question taboue, pratique honteuse. Si l'on sanctionnait, il ne fallait surtout pas en parler. La sanction a parfois été considérée comme une question secondaire, comme si finalement le cœur du travail éducatif se trouvait ailleurs. Et pourtant, qui ne voit que penser la sanction, c'est toujours penser beaucoup plus qu'elle. C'est déjà apprécier le cadre socialisant que l'on offre aux enfants ou aux élèves, c'est aussi interroger les postures et les attitudes de ceux qui ont la responsabilité de les éduquer. Défaite de la pensée, négation de l'éducation, ces illusions se sont par bonheur bien souvent dissipées.

Il faut être foucaldien en la matière et accepter l'idée qu'il y a une heuristique de la marge. Que ce n'est pas en décryptant les grands et beaux principes, inscrits en lettres d'or au frontispice des institutions éducatives, que l'on comprend ce qu'éduquer veut dire, à un moment donné, pour une société donnée. Mais que c'est examinant ce que l'on pourrait appeler « la banlieue du travail éducatif ». Primauté de la périphérie, primat des marges sur le cœur. La sanction nous invite à nous intéresser à ces pratiques éducatives qui menacent toujours de devenir autres : brimades, violences, coercition... Il faut oublier les belles déclarations de circonstance et savoir observer les réalités peu glorieuses de tous les jours; mettre en avant non ce que les éducateurs ont aimé dire mais ce qu'ils ont accepté de faire. Il est maintenant temps de commencer notre voyage, partons pour l'Afrique, direction le Burkina Faso et le Bénin.

L'article sur l'école burkinabè montre toute la difficulté que peut avoir une institution pour rompre avec des pratiques installées de longue date. Des enseignants pas toujours convaincus qu'il existe d'autres possibles, des parents qui oscillent entre défense et condamnation des châtiments corporels, de jeunes organisations non gouvernementales qui en appellent au respect des droits de l'enfant, l'article des professeurs Soré et Moyenga illustre ce qu'il convient d'appeler une période de transition. «Entre interdiction, survivance et innovation» titrent nos auteurs. Cette 
contribution met aussi en lumière la puissance toute relative du droit car si l'interdiction des châtiments corporels au Burkina Faso remonte à 1962, la « chicotte » (sorte de fouet) est encore bien présente dans les lieux d'enseignement. Cette situation n'est pas propre au Burkina Faso. En France, l'interdiction des châtiments, déjà lointaine (1803), ne sera vraiment effective que dans la seconde moitié du xx siècle. Inévitable décalage car on ne peut tourner la page de la violence éducative que si s'esquissent d'autres manières de faire. "Lorsque l'on veut changer les mœurs et les manières, notait déjà Montesquieu, il ne faut pas les changer par les lois, cela paraîtrait trop tyrannique : il vaut mieux les changer par d'autres mœurs et d'autres manières » (Montesquieu, 2013). Le droit a bien évidemment des vertus mais il ne faut pas les surestimer. Il soutient les changements plus qu'il ne les initie, il accompagne les pratiques plus qu'il ne les promeut.

Dans le Bénin voisin, les autorités éducatives sont elles aussi confrontées à la persistance des sévices corporels, alors que ceux-ci sont, comme au Burkina Faso, explicitement prohibés depuis plusieurs années déjà. La même résistance, les mêmes réticences... Mais l'article de Clarisse Napporn éclaire une autre facette de la réalité punitive : le recours aux pratiques humiliantes. Ou quand punir, c'est faire honte. Or, si la honte est l'épreuve de la mésestime de soi, alors n'est-ce pas encore faire mal ? "La honte est un sentiment terrible, écrit Serge Tisseron, parce que celui qui l'éprouve craint d'être définitivement exclu du groupe dont il fait partie » (Tisseron, 2006). La honte désocialise. Pas toujours, répond le pénaliste australien John Braithwaite, qui soutient, dans un ouvrage récent, qu'il existe une « honte réintégratrice » (reintegrative shame). La honte aurait des vertus socialisantes si elle stigmatise la seule infraction (Braithwaite, 1989). Indignité de l'acte et non de la personne. D'où le retour de la honte dans certains systèmes juridiques modernes, retour d'un affect que l'on croyait pourtant d'un autre temps.

Dans la seconde partie de l'article, C. Napporn se met à l'écoute des élèves et des parents. Comment les élèves perçoivent-ils les sanctions qu'on leur applique? Comment les parents jugent-ils ces pratiques? Les parents, nous l'auteure, admettent dans leur très grande majorité l'idée que l'on puisse sanctionner des élèves mais ils demandent dans le même moment plus de dialogue et de compréhension de la part des professeurs. Il faut, disent en substance ces parents, encore et toujours parler. Et l'on ne peut s'empêcher de penser qu'ils ont raison car la sanction appelle et exige la parole. Au plan du sens, la parole relie la sanction à la transgression, elle établit une sorte de pont symbolique ; au plan pragmatique, elle les dissocie en introduisant quelques mots entre la bêtise et la réponse punitive. La parole lie et délie. On peut même dire que c'est parce que la vengeance est l'acte silencieux par excellence que la sanction s'annonce, se parle, et pour tout dire s'explique.

Le texte d'Hiroshi Nakada nous emmène au Japon. Son propos sur les châtiments fait écho, par certains aspects, aux contributions de nos collègues africains. On pense aussi à ce qui s'est passé et se passe aujourd'hui encore dans certains pays européens ou États américains, et on ne peut manquer de se dire que les châtiments corporels ont été pratiqués sous toutes les latitudes. Frapper les enfants a été, il faut hélas le reconnaître, une pratique quasi universelle. Avec, étonnante coïncidence, 
au Japon comme ailleurs, toujours le même instrument. Il y aurait une histoire du fouet à écrire. Il n'était pas rare en Europe, au Xvi siècle, que l'on remette au professeur, le jour de son installation, un fouet pour le consacrer dans l'exercice de sa nouvelle fonction. Ce n'est que beaucoup plus tard que le livre imposera son prestige pour se substituer au fouet comme symbole de la régence. Mais le Japon n'est ni l'Afrique ni l'Europe car, comme le précise Hiroshi Nakada, il a existé très tôt, au pays du Soleil-Levant, dès le viII ${ }^{\mathrm{e}}$ siècle, des moines bouddhistes réfractaires à toutes formes de châtiments physiques. L'auteur a raison d'insister sur cet aspect proprement exceptionnel, car cela n'a été nullement le cas dans les sociétés christianisées qui n'ont cessé de plaider pour une souffrance rédemptrice.

Le mot «verge » apparaît d'ailleurs pas moins de cinquante fois dans l'Ancien Testament, ce qui fait dire à l'auteur de l'article «Punitions » du dictionnaire Buisson que « l'Ancien Testament est certainement de tous les livres sacrés [...] celui où il est fait le plus mention de châtiments » (D'Arvert, 1887). Ce n'est qu'avec les encyclopédistes que l'étrange lien douleur/vertu sera définitivement dénoué (Diderot, 1765). Mais il est encore une chose importante à noter dans ce texte sur les châtiments au Japon, c'est le lien que fait l'auteur entre la dégradation générale d'une situation politique et les risques de dérives en matière éducative. Comme si l'atmosphère sociétale ambiante avait une incidence directe sur la manière dont on éduquait les enfants. «Le Japon à cette époque, note Hiroshi Nakada, était en guerre et il est possible que les châtiments corporels aient été pratiqués au sein de familles afin d'éduquer et de former de futurs samouraïs ». Les formes punitives, et de manière générale les pratiques éducatives, ne sont jamais en apesanteur, elles s'inscrivent toujours dans un contexte socio-politique qui les détermine en partie.

Avant de nous rendre en France, faisons un petit détour par la Russie. Celui qui s'est un peu intéressé aux pratiques punitives dans les pays d'Europe occidentale ne sera guère dépaysé. Car à côté des traditionnels châtiments corporels, écrivent Elena Nikolaeva et Lyudmila Savinova, d'autres types de sanctions furent introduits, comme la stigmatisation, la mise à l'écart ou l'isolement " en cellule disciplinaire ». Rien de nouveau sous le soleil de l'est. On y apprend encore que les châtiments corporels seront formellement abolis en 1864 avec la nouvelle loi sur les collèges et lycées, et que les dirigeants de l'Église orthodoxe s'opposeront avec vigueur à une telle libéralisation. Rien encore de bien surprenant.

Mais la Russie a eu tout au long du siècle dernier, ne l'oublions pas, de grands éducateurs confiants en l'enfance. Ce texte rend justice à l'un de ceux-ci : Vasyl Sukhomlinsky (1918-1970). On sait l'importance de Tolstoï et de Makarenko, mais on regrette parfois que ces illustres noms, déjà consacrés, captent toute la lumière, laissant dans l'ombre des éducateurs hors norme dont Vasyl Sukhomlinsky fait assurément partie. Il publie en 1969, un peu avant de s'éclipser, son ouvrage testament, Aux enfants, je donne mon cour, dans lequel il prône une éducation bienveillante sachant tenir à distance toutes les formes de la maltraitance et de l'humiliation. "Je suis fermement convaincu, écrivait-il, qu’il existe des qualités d'âme sans lesquelles une personne ne peut devenir un véritable éducateur, et parmi celles-ci, la capacité d'entrer dans le monde intérieur d'un enfant occupe une place de choix » (cité par A. Cockerill, 2001). Sukhomlinsky ou l'éloge de l'empathie avant l'heure. 
En route maintenant vers la France. Le propos sociologique de Sylvie Ayral s'ouvre sur un constat : $80 \%$ des élèves punis dans les collèges français sont des garçons. Loin d'accepter ce fait comme allant de soi, l'auteure l'interroge car cette surreprésentation masculine inquiète tout autant qu'elle pose question. L'article montre, empirie à l'appui, que le système punitif français participe à la construction et à la reproduction des inégalités sexuées. La sanction est vécue par certains élèves comme une épreuve où se nouent défi et affirmation viriliste. Plus encore, la sanction rend visible une hiérarchie de genre à l'intérieur même du monde des garçons "Ceux qui sont punis, les dominants violents, sexistes et homophobes présentent des comportements conformes aux normes de la virilité, c'est-à-dire au sexe prescrit. Les autres sont déclassés dans la catégorie des sous-hommes, c'est-à-dire des faibles, des pédés et deviennent les cibles potentielles des premiers ». Mais ce qui surprend le plus dans cette contribution, c'est d'apprendre que certains professeurs contribuent par leur comportement à maintenir cet imaginaire d'une sexualité dominante en laissant penser qu'ils sont prêts à en découdre physiquement.

"La sanction confère finalement, conclut Sylvie Ayral, une importance chaque jour renouvelée au Masculin : à l'autorité masculine mais aussi aux garçons punis, qu'ils soient désignés comme des modèles masculins négatifs par l'institution ou comme des modèles masculins positifs par la communauté des pairs ». Il faudrait montrer que cette éducation genrée est initiée bien avant l'école, lorsque les familles posent les toutes premières pierres du travail éducatif. Nous avons encore en tête le programme ABCD de l'égalité, proposé en 2013 pour lutter contre le sexisme et les stéréotypes de genre, programme qui sera vivement contesté, non par les professeurs, mais par les familles. Les contributions rassemblées dans le présent dossier sont centrées sur l'école, il faudrait consacrer un numéro à l'exploration des pratiques éducatives et punitives des familles. Nous serions sans doute surpris de découvrir que la famille, contrairement à ce que l'on peut parfois penser, est toujours un lieu plus violent et maltraitant que l'école.

Avec la contribution de Rosa Mahdjoub et Mohamed Miliani, nous traversons la Méditerranée. Leur texte décrit une école algérienne si ce n'est en plein tumulte tout au moins désemparée. Des élèves plus difficiles, des professeurs contrariés pour ne pas dire un peu perdus et des parents moins enclins à les soutenir, tels sont les principaux éléments du tableau. La sanction dans nombre de pays, et l'Algérie sur ce point ne fait pas exception, est très souvent la première cause de divorce entre parents et enseignants. Divorce qui va parfois jusque devant les tribunaux. Mais l'important est ailleurs, il tient en une date : juillet 2018. Fait étonnant, ce ne sont pas moins de dix arrêtés ministériels qui vont être promulgués pour tenter de stabiliser les pratiques punitives aux différents niveaux de l'institution scolaire. Le succès ne sera pas au rendez-vous car le défi de l'école algérienne est ailleurs. Ce sont aujourd'hui deux cultures qui s'affrontent, une culture laïque, partiellement disqualifiée car héritée de la période coloniale, qui parle en termes de lois et de citoyenneté, et une culture religieuse, qui pose les problèmes en termes de modèle et de vertus. Le mérite de cette contribution est de nous montrer que toute conception punitive cohérente requiert un horizon éducatif partagé. Elle présuppose plus fondamentale- 
ment un accord sur le sens du travail éducatif. Pour le dire autrement et plus simplement : que doit-on viser quand on sanctionne un écolier dans une école, un collégien dans un collège, un lycéen dans un lycée et, plus généralement, un enfant dans une institution qui se veut éducative ? Périlleuse question que celle des fins, mais aucune école, aucun système éducatif ne saurait l'esquiver.

Cap vers l'Angleterre et l'optimisme avec le texte de Dame Alison Peacock. $\mathrm{Ou}$ comment transformer une école au bord du chaos en un lieu où les élèves retrouvent l'envie d'apprendre et les enseignants le plaisir d'enseigner. L'auteure, aujourd'hui présidente du Chartered College of Teaching, narre son expérience à la direction de la Wroxham School de 2004 à 2016. Elle décrit minutieusement les techniques qu'elle a mises en œuvre pour redonner envie et confiance aux élèves. Comme par exemple "les réunions de cercle ", ces modes de fonctionnement " restauratifs » qui privilégient l'échange et la responsabilité. Dame Alison Peacock souligne également l'importance de l'encouragement et de la valorisation, nous rappelant au passage que le terme de sanction a bel et bien deux valences. Celle-ci peut certes être entendue comme une punition mais elle doit aussi être comprise comme une récompense. On lira avec fruit le paragraphe intitulé " accompagner et réguler les comportements ». Il met en évidence que la sanction ne peut être qu'un élément d'un projet éducatif plus ample qui subsume cette dernière dans une visée socialisante. Mais le principe phare de la Wroxham School est sans doute d'avoir toujours refusé de cataloguer, de noter ou de classer les élèves. Il y a du Rousseau dans cette expérimentation. "Il est bien étrange, se désolait le philosophe genevois, que, depuis qu'on se mêle d'élever des enfants, on n'ait imaginé d'autre instrument pour les conduire que l'émulation, la jalousie, l'envie, la vanité, l'avidité, la vile crainte, toutes les passions les plus dangereuses (...) et les plus propres à corrompre l'âme » (Rousseau, 1951, Livre II).

Pour bien comprendre la contribution américaine qui fait suite et prolonge le propos de Dame Alison Peacock, il faut avoir quelques lumières sur ce que l'on appelle la justice restaurative. Nous avons déjà lâché le mot, il faut maintenant en dire plus. De quoi s'agit-il précisément ? Cette conception juridique est née dans les années soixante-dix (1970) en Amérique du Nord. Elle part d'un double constat critique à l'endroit des justices rétributives classiques. Le premier est que ces dernières négligent le travail de resocialisation des infracteurs. Une fois la peine accomplie, ces derniers retournent comme si de rien n'était à leurs activités. Le second est l'absence de prise en charge des victimes. Et s'il en est ainsi, c'est parce que les justices rétributives conçoivent l'infraction d'abord comme une atteinte à la majesté de la Loi alors qu'elle est, pour les tenants d'une approche restaurative, un ensemble de torts qui affectent des personnes et au-delà... une communauté. Car, comme l'écrit Howard Zehr, le père de la justice restaurative, « le crime a un impact sur les communautés et dans bien des cas, celles-ci devraient être considérées comme parties prenantes en tant que victimes secondaires» (Zehr, 2012, c'est nous qui soulignons).

La question finalement est moins « qui est l'infracteur? » que " quels sont les torts qui ont été commis ? ». L’approche restaurative met alors légitimement l'accent sur la réparation des dommages (physiques, psychiques, matériels...) ainsi 
que sur la restauration des liens sociaux un instant maltraités par l'infraction. «To put things right " : réparer et retrouver l'équilibre. On comprend dès lors pourquoi la justice restaurative préconise des dispositifs qui invitent à la rencontre entre victimes et infracteurs, rencontres qui sont toujours des moments où les torts et les responsabilités sont établis (cercle, conférence restaurative...). On comprend aussi pourquoi la justice restaurative invite à mettre en œuvre des procédures réparatoires. On comprend, enfin, pourquoi elle favorise les prises de décision partagées. Car la manière dont on sanctionne conditionne toujours, qu'on le veuille ou non, l'avenir de ceux qui auront à revivre ensemble. C'est à l'aune de ces principes qu'il faut lire la contribution de Suzanne Hitchman et Anne Gregory et leur souci d'introduire des pratiques restauratives au sein de l'école. Ce texte est aussi une invitation à méditer sur les promesses et les limites de telles pratiques.

Dernière escale : le Québec. "La régulation des comportements, note Denis Jeffrey, s'est complexifiée (...) avec la massification scolaire au cours des années 1960-1970, avec la diversification des populations scolaires venues de l'immigration et avec l'inclusion des élèves qui souffrent de problèmes de santé, de comportement et d'apprentissage dans les classes ordinaires. La gestion de classe, même pour les enseignants les plus expérimentés, est devenue un lourd fardeau ». Enseigner au Québec, comme dans bien d'autres sociétés, est devenu un art difficile. Les enseignants sont aujourd'hui confrontés à un terrible défi, non celui de la violence comme on n'a cessé de le répéter, mais celui de l'indiscipline chronique. Si dans les années qui suivent la Révolution tranquille, une forte majorité d'enseignants québécois se tourne vers les solutions de la pédagogie positive, cette même majorité semble aujourd'hui déchanter. «Ils ont adopté la pédagogie positive, écrit Denis Jeffrey, mais ils sont de plus en plus nombreux à reconnaître ses imperfections et l'importance d'assumer une position d'autorité professionnelle auprès des élèves ».

Il y a comme un rêve oublieux de l'histoire qui revient sans cesse : celui d'une école sans contrainte et sans sanction. Et pourtant, rappelons-nous Hambourg et ses écoles libertaires (1920). "Dès les premiers jours, écrit Schmid qui rapporte cette étonnante expérience, les maîtres annoncèrent à leurs élèves qu'il n'existerait plus de punition ni de sanction, qu'il ne serait plus question d'interdiction ou d'un règlement quelconque qui pourrait les gêner dans l'usage de leur pleine liberté » (Schmid, 1972). Or il fallut un jour se rendre à l'évidence. Échec d'autant plus amer que pendant plus de dix ans, ces maîtres novateurs avaient fait preuve d'une énergie et d'un enthousiasme peu communs. Zeidler, l'un des inspirateurs du projet, dut reconnaître non sans tristesse, après plusieurs années d'expérience, que "partout où l'on se laissa guider par une confiance sans borne dans le tact des enfants, dans leur force de volonté, dans leur persévérance, dans la sûreté de leur instinct et dans la tolérance des individus à former une communauté (...), on vit se former des bandes d'indisciplinés » (Schmid, 1972).

On a écrit depuis bien longtemps déjà l'histoire du maître sadique et punisseur, il y aurait à écrire, en contre-point, une autre histoire, plus contemporaine, celle du maître qui rechigne à sanctionner. "Lorsqu'il s'agit d'une classe, confesse Florence Ehnuel, écrivaine et professeur de philosophie en lycée, les sanctions m'ap- 
paraissent donc aujourd'hui comme le préalable à un comportement plus responsable. Elles doivent être réfléchies par l'enseignant, jamais humiliantes, et aussi justes que possible ; elles peuvent aussi être commentées avec l'élève concerné. Mais je ne pense plus pouvoir en faire l'économie. Je décèle même maintenant dans la répulsion que j'ai longtemps éprouvée à les appliquer une forme de lâcheté » (Florence Ehnuel, 2012). Dans cette histoire, il y aurait un chapitre à consacrer à la peur de sanctionner qui n'est autre que la peur d'abîmer la relation. La rupture est l'angoisse par excellence du pédagogue. Maintenir le contact, garder la confiance, voilà la condition première de toute action éducative. Qu'importe le plan, l'objectif ou encore l'intention théorique, si autrui se dérobe, le plus beau des projets éducatifs est déjà compromis.

Mais la confiance emprunte souvent des chemins que l'on ne soupçonne pas. Maria Teresa Estrela, professeure de psychologie à l'université de Lisbonne, écrivait dans le milieu des années quatre-vingt-dix (1990) : "plus les élèves sont indisciplinés, plus ils font la louange des enseignants capables de maintenir l'ordre » (Estrela, 1994). Alain le disait déjà : «Et sans exception, ceux qui voulaient persuader, écouter, dissiper, pardonner enfin aux promesses, étaient méprisés, hués, et, chose triste à dire, finalement haïs ; au lieu que les autres, les hommes sans cour, étaient finalement aimés»(Alain, 1957). Mais ne nous méprenons pas sur ces «sans cœur ", ce sont des professeurs exigeants, déjà exigeants avec eux-mêmes, et toujours soucieux de justice. Qu'est-ce que je me permets, moi professeur ? Qu'est-ce que je ne me permets pas ? Comment être juste sans céder à la complaisance ? Questions d'éthique.

Il ne s'agit pas bien évidemment de balayer d'un revers de main les innovations. Les intuitions de la justice restaurative nous interpellent. Mais nous ne saurions non plus oublier qu'au principe de toute socialisation est la règle à respecter. Car, comme le dit encore Alain, "il y a toujours désordre en une réunion d'élèves qui n'ont point coutume d'être ensemble » (Alain, 1957). La bonne voie consiste sans doute à penser des formes hybrides qui sachent articuler rétribution et restauration. Le philosophe Conrad Brunk nous a montré qu'il ne fallait plus les opposer comme on oppose le bien et le mal (Brunk, 2001). Howard Zehr le reconnaît lui-même aujourd'hui. « Dans mes écrits précédents, note le criminologue américain, je traçais une frontière nette entre une justice pénale à caractère rétributif et une approche restaurative de la justice. Ces derniers temps toutefois, j'en suis venu à penser que cette polarisation peut s'avérer trompeuse. S’il est possible de tracer un schéma qui mette en avant le contraste entre les deux approches, son formalisme empêche de voir ce qui les rapproche et les modes de collaboration qui peuvent s'établir entre elles » (Zehr, 2012).

La question de la sanction, longtemps oubliée, souvent passée sous silence, est bel et bien une question éducative majeure. Kant, dans ses Réflexions sur l'éducation expliquait, il y a plus de deux siècles déjà, que la contrainte n'est pas contraire à l'action éducative. Éduquer, disait-il, c'est libérer mais on ne peut rendre libre que si l'on se sert de la contrainte. «Un des grands problèmes de l'éducation est le suivant : comment unir la soumission sous une contrainte légale avec la faculté de se servir de sa liberté ? Car la contrainte est nécessaire ! Mais comment puis-je cultiver la liberté 
sous la contrainte ? Je dois habituer mon élève à tolérer une contrainte pesant sur sa liberté, et en même temps, je dois le conduire lui-même à faire un bon usage de sa liberté. Sans cela tout n'est que pur mécanisme et l'homme privé d'éducation ne sait se servir de sa liberté » (Kant, 1989, Introduction).

Ce propos n'a pas pris une ride car toute la difficulté, tout le sel de la tâche éducative réside dans ce paradoxe : comment se servir de la contrainte pour rendre l'autre libre ? Il nous faut aujourd'hui renouveler le geste de Kant, savoir réconcilier éducation et sanction et montrer que cette dernière n'est pas vouée à être une parenthèse ou un artéfact dans le procès éducatif mais qu'elle peut en devenir sous certaines conditions un moment dynamique et positif. Tel est notre défi pour les années à venir.

\section{BIBLIOGRAPHIE}

ALAIN (1957). Propos sur l'éducation. Paris : Presses universitaires de France.

BRAITHWAITE J. (1989). Crime, Shame and Reintegration. Cambridge : Cambridge Press University.

BRUNK C. (2001). « Restorative Justice and the Philosophical Theories of Criminal Punishment » in M. L. HADLEY (ed.). The Spiritual Roots of Restorative Justice. New-York : State University of New York Press, p. 31-56.

COCKERILL A. (2001). «The Educational philosophy of V. A. Sukhomlinski ». En ligne : [http://www.sote.qld.edu.au/articles/Sukhomlinsky.html], consulté le 2 octobre 2019.

D'ARVERT F. (1887). « Punitions ", in Dictionnaire de pédagogie et d'instruction primaire, sous la direction de F. Buisson. Paris : Hachette \& Cie, $1^{\text {re }}$ partie, t. II, p. 2491-2494.

DIDEROT D. (1765). "Châtiment ", in Encyclopédie ou dictionnaire raisonné des sciences, des arts et des métiers. Paris, t. I, p. 250.

EHNUEL F. (2012). Le bavardage, parlons-en enfin. Pour une classe à l'écoute. Paris : Fayard.

ESTRELA M. T. (1994). Autorité et discipline à l'école. Paris : ESF.

KANT E. (1989). Réflexions sur l'éducation. Paris : Vrin.

MONTESQUIEU (2013). De l'esprit des lois. Paris : Flammarion.

ROUSSEAU J.-J. (1951). Émile ou de l'éducation. Paris : Garnier.

TISSERON S. (2006), « De la honte qui tue à la honte qui sauve ». Le Coq-Héron, 2006/1, $\mathrm{n}^{\circ} 184$, p. $18-31$.

ZEHR H. (2012). La justice restaurative. Pour sortir des impasses de la logique punitive. Genève : Labor et Fides (Le champ éthique). 